\title{
Ethanol Thymus serpyllum extracts: evaluation of extraction conditions via total polyphenol content and radical scavenging activity
}

\author{
Aleksandra Jovanović ${ }^{1, *}$, Mihaela Skrt², Predrag Petrović ${ }^{1}$, Ivona Častvan ${ }^{1}$, Gordana \\ ZDUNIĆ $^{3}$, KATARINA ŠAVIKIN ${ }^{3}$, AND BRANKO BUGARSKI ${ }^{1}$ \\ ${ }^{1}$ University of Belgrade, Faculty of Technology and Metallurgy, Karnegijeva 4, 11000 Belgrade, Serbia \\ ${ }^{2}$ University of Ljubljana, Department of Food Science and Technology, Biotechnical Faculty, Jamnikarjeva 101, 1000 Ljubljana, Slovenia \\ 3 Institute for Medicinal Plant Research "Dr Josif Pančić", Tadeuša Košćuška 1, 11000 Belgrade, Serbia \\ *Corresponding author: acancarevic@tmf.bg.ac.rs
}

Received: October 28, 2019

Accepted: November 11, 2019

Published on-line: November 29, 2019

Published: December 25, 2019

\begin{abstract}
The aim of the present study was the optimization of the extraction conditions (particle size, solidto-solvent ratio, extraction time and procedure) via total polyphenol content and radical scavenging activity, using wild thyme (Thymus serpyllum L.) as a natural source of polyphenols. According to the total polyphenols yield, particle size, solvent-to-solid ratio, and extraction technique have shown statistically significant influence, whereas the exposure time has not been a relevant factor. In all three extraction procedures (maceration, heat- and ultrasound-assisted extraction), the highest polyphenols yield was reached using the smallest plant particles and the highest solvent-to-solid ratio. Moreover, polyphenols content was significantly higher after ultrasound-assisted extraction, in comparison to the extraction at room and high temperature. According to the antioxidant recovery, the influence of different extraction parameters varied depending on the applied antioxidant tests and extraction procedures. 2,2'-azino-bis-(3-ethylbenzothiazoline-6-sulfonic acid) - ABTS radical scavenging capacity of extracts followed the next trend: maceration and ultrasound-assisted extraction > heat-assisted extraction, while in 2,2-diphenyl-1-picrylhydrazyl - DPPH neutralization: ultrasound-assisted extraction > heat-assisted extraction $>>$ maceration. The study represents an important step in the formulation of antioxidant-rich extracts with potential use in food, pharmaceutical, and cosmetic industry.
\end{abstract}

Key words: extraction, wild thyme, total polyphenols, radical scavenging activity

http://dx.doi.org/10.5937/leksir1939023J

\section{INTRODUCTION}

Polyphenols, presented in various natural sources, possess numerous biological effects, such are antioxidant, anticarcinogenic, anti-inflammatory, gastroprotective, antiallergic and antimicrobial properties (Boros et al., 2010; Nikolić et al., 2014; Spormann et al., 2008). Therefore, polyphenol extracts can be used as an ingredient in the production of healthy or functional foods and as an active component in the pharmaceutical products for potential treatment of different gastrointestinal, cardiovascular, respiratory and metabolic diseases (González et al., 2019; Hosseinzadeh et al., 2015). Moreover, polyphenol compounds, as plants' secondary metabolites, can be employed as food preservatives and antioxidants, particularly in meat and fish (Casiglia et al., 2015). Thus, with the aim to produce a healthy, natural and low-cost alternative to synthetic additives that can manifest some toxic effects, different polyphenol extraction techniques were estimated (Čanadanović-Brunet et al., 2006). Considering the great structural diversity of polyphenol compounds, it is important to optimize extraction conditions in order to extract maximal yield of target substances from a certain plant source (Jovanović et al., 2017b). The aim of the presented study was the examination of the influence of different extraction conditions: (1) particle size $(0.3,0.7$ and $1.5 \mathrm{~mm}),(2)$ solvent-to-drug ratio $(10: 1,20: 1$ and 30:1), (3) extraction procedure (maceration, heatand ultrasound-assisted extraction) and (4) time (depending on the applied extraction technique) on total polyphenols content and radical scavenging activity. This work represents the expansion of previously published study (Jovanović et al., 2017a), with the aim to investigate different Thymus serpyllum extracts obtained by using absolute ethanol. 


\section{MATERIALS AND METHODS}

\subsection{Plant material and chemicals}

Air dried and grounded wild thyme herb was obtained from the Institute for Medicinal Plant Research "Dr. Josif Pančić", Serbia, and used as a plant source for polyphenol extraction. Ethanol and sodium carbonate were purchased from Fisher Scientific, UK; Folin-Ciocalteu reagent and gallic acid from Merck, Germany; potassium persulfate from Centrohem, Serbia; 2,2'-azino-bis-(3-ethylbenzothiazoline-6-sulfonic acid) - ABTS, 6-hydroxy-2,5,7,8-tetramethylchroman-2-carboxylic acid - Trolox and 2,2-diphenyl-1-picrylhydrazyl - DPPH from Sigma-Aldrich, USA.

\subsection{Extraction}

The extraction was carried out using (1) three sizes of plant particles $(0.3,0.7$ and $1.5 \mathrm{~mm}),(2)$ three solvent-to-solid ratios (10:1, 20:1 and 30:1), and (3) five extraction times in the case of maceration $(5,15,30,60$ and $90 \mathrm{~min})$ and three extraction times in the case of heat- and ultrasound-assisted extraction $(5,15$ and $30 \mathrm{~min})$, while absolute ethanol was used as an extraction solvent. Three different extraction procedures (maceration, heat- and ultrasound-assisted extraction) were used and compared as well. Maceration was performed using Unimax 1010 air shaker (Heidolph, Germany) at $25^{\circ} \mathrm{C}$, whereas heat-assisted extraction was carried out in the incubator shaker KS 4000i control, (IKA, Germany) at $80^{\circ} \mathrm{C}$. The ultrasound processor $(750 \mathrm{~W}$, Sonics, USA) with a converter $(20 \mathrm{kHz})$ and a solid titanium probe (diameter of $19 \mathrm{~mm}$ ) were used for ultrasound-assisted extraction, at amplitude of $80 \%$.

\subsection{Total polyphenol content}

Total polyphenol content was determined according to the previously used Folin-Ciocalteu procedure (Skotti et al., 2014) Ethanol extracts (properly diluted, $20 \mu \mathrm{L}$ ), Folin-Ciocalteu reagent water solution $(100 \mu \mathrm{L}, 1: 2 \mathrm{v} / \mathrm{v})$, sodium carbonate water solution $(300 \mu \mathrm{L}, 20 \% \mathrm{v} / \mathrm{v})$ and distilled water $(1.58 \mathrm{~mL})$ were mixed and the absorbance was measured after $2 \mathrm{~h}$ at 765 $\mathrm{nm}$, using UV spectrophotometer UV-1800 (Shimadzu, Japan). The calibration curve was calculated using gallic acid as a standard (100-700 mg/L), while the results were presented as $\mathrm{mg}$ of gallic acid equivalents per $\mathrm{g}$ of drug (mg GAE/g).

\subsection{Radical scavenging activity}

\subsubsection{ABTS assay}

The assay was carried out using the procedure described by Petrović et al. (2016), ABTS ${ }^{+}$free radicals were generated by mixing potassium persulfate water solution $(3.8 \%, 88 \mu \mathrm{L})$ and $\mathrm{ABTS}^{+}$water solution $(0.38 \%, 5 \mathrm{~mL})$ and keeping the reaction mixture during $24 \mathrm{~h}$ at $4{ }^{\circ} \mathrm{C}$. ABTS ${ }^{+}$solution was diluted using pure ethanol in order to achieve the absorbance value of $0.700 \pm 0.02$. Diluted solutions of the extracts $(20 \mu \mathrm{L})$ were added in $\mathrm{ABTS}^{++}$solution $(2 \mathrm{~mL})$ and the absorbance readings $\left(\mathrm{A}_{\mathrm{s}}\right)$ were taken after $6 \mathrm{~min}$ at $734 \mathrm{~nm}$. A mixture of ABTS ${ }^{+}$ solution $(2 \mathrm{~mL})$ and pure ethanol $(20 \mu \mathrm{L})$ was used as a control $\left(\mathrm{A}_{\mathrm{c}}\right)$. $\mathrm{ABTS}^{+}$scavenging activity was calculated using the following equation:

$$
\Delta \mathrm{A}=A_{c}-A_{s}
$$

The calibration curve was calculated using Trolox as a standard $(0.2-1.0 \mathrm{mM})$, and the results were expressed as $\mathrm{mmol}$ Trolox equivalents per milliliter of extract ( $\mathrm{mmol}$ Trolox $/ \mathrm{mL}$ ).

\subsubsection{DPPH assay}

The antioxidant capacity of wild thyme extracts was measured in terms of hydrogen donating or radical scavenging activity, using the stable DPPH radical (Petrović et al., 2019). All samples were prepared as follows: $200 \mu \mathrm{L}$ of five different concentrations of ethanol extract was mixed with $2.8 \mathrm{~mL}$ of the DPPH radical solution (DPPH powder dissolved in ethanol) and the absorbance (As) was read after $20 \mathrm{~min}$ at $517 \mathrm{~nm}$. A mixture of DPPH ethanol solution $(2.8 \mathrm{~mL})$ and pure ethanol $(200 \mu \mathrm{L})$ was used as a control $\left(\mathrm{A}_{\mathrm{c}}\right)$. The antioxidant ability was calculated as follows:

$$
\text { Radical Scavenging Capacity }(\%)=\frac{A_{c}-A_{s}}{A_{c}} \times 100 \%
$$

The results were expressed as $\mathrm{IC}_{50}(\mathrm{mg} / \mathrm{mL})$, the concentration of extract required to scavenge $50 \%$ of free radicals.

\subsection{Statistical analysis}

All extraction procedures, measurements and analyses were done in triplicate. The statistical analysis was carried out using the following statistical tools: analysis of variance (oneway ANOVA), Duncan's test, within the statistical software, STATISTICA 7.0. The data in table and graphs are presented as mean \pm standard deviation, whereas the differences were considered statistically significant at $\mathrm{P}<0.05$. Independent variables were particle size, solvent-to-solid ratio, extraction procedure and time, while total polyphenols content and radical scavenging activity were dependent variables. The effects and corresponding regression coefficients of factors and factor interactions (within the first factorial design) were observed as well.

\section{RESULTS AND DISCUSSION}

\subsection{Total polyphenol content}

The influence of the particle size, solvent-to-solid ratio and time, using different procedures (maceration, heat- and ultrasound-assisted extraction), on the polyphenols yield was investigated. The contents of extracted polyphenols for all tested conditions are presented in Table 1.

\subsection{Maceration}

Particle size is one of the important factors affecting the extraction yield. Thus, the effect of plant particle size $(0.3,0.7$ and $1.5 \mathrm{~mm}$ ) on the total polyphenol amount in maceration is presented in Table 1. According to the results, there was a statistically significant difference in phenolic content depending on the particle size level. Polyphenol yield was significantly higher $(\sim 60-75 \%)$ using $0.3 \mathrm{~mm}$ particles, compared to the other two levels of particle size. Boros et al. (2010) showed that the reduction of particle size (degradation of plant cell and tissue, respectively), as well as the increasing of the surface area led to the faster mass transfer and thus higher extraction yield. The presented data are in agreement with previous research which showed that the greatest yield of polyphenols was obtained using the highest degree of grinding (Jovanović et al., 2017b).

Three solvent-to-solid ratios (10:1, 20:1 and 30:1) were selected to evaluate the impact of the extraction solvent amount on the total polyphenol content. In the maceration, there was no statistically significant difference between 20:1 and 30:1 ratios, whereas 10:1 ratio gave significantly lower polyphenol content (Table 1). Zdravković et al. (2012) also showed that solvent:drug ratio increase above 15:1 only slightly increased the polyphenol extraction yield, in case of maceration of nettle leaves. However, previous studies have reported that the extraction of polyphenols from natural sources is significantly higher when larger quantities of extraction medium are used (at room temperature); this prevents the extraction medium' saturation and thus enhances polyphenols release (Jovanović et al., 2017b). 
Table 1. Total polyphenol content in wild thyme (Thymus serpillum) extracts obtained using different extraction procedures

\begin{tabular}{|c|c|c|c|c|}
\hline \multirow[b]{2}{*}{ Factor } & \multirow[b]{2}{*}{ Level } & \multicolumn{3}{|c|}{ Total polyphenols content ${ }^{\mathrm{a}}$} \\
\hline & & Maceration & Heat-assisted extraction & Ultrasound-assisted extraction \\
\hline & & [mg GAE/g] & [mg GAE/g] & [mg GAE/g] \\
\hline \multirow[t]{3}{*}{ Particle size (mm) } & 0.3 & $4.27 \pm 0.57 \mathrm{a}$ & $7.08 \pm 1.01 \mathrm{a}$ & $7.04 \pm 1.03 \mathrm{a}$ \\
\hline & 0.7 & $2.72 \pm 0.40 \mathrm{~b}$ & $5.88 \pm 0.73 b$ & $5.61 \pm 0.75 b$ \\
\hline & 1.5 & $2.45 \pm 0.37 b$ & $4.55 \pm 0.46 \mathrm{c}$ & $5.51 \pm 0.70 b$ \\
\hline \multirow[t]{3}{*}{ Solvent-to-solid ratio } & $10: 1$ & $2.54 \pm 0.58 b$ & $4.16 \pm 0.54 b$ & $4.48 \pm 0.39 b$ \\
\hline & $20: 1$ & $3.40 \pm 0.55 a$ & $5.42 \pm 0.68 \mathrm{ab}$ & $6.72 \pm 0.56 \mathrm{a}$ \\
\hline & $30: 1$ & $3.50 \pm 0.60 \mathrm{a}$ & $6.93 \pm 0.99 a$ & $7.66 \pm 0.61 \mathrm{a}$ \\
\hline \multirow[t]{5}{*}{ Time (min) } & 5 & $2.68 \pm 0.54 b c$ & $5.19 \pm 1.12 \mathrm{a}$ & $5.93 \pm 0.98 \mathrm{a}$ \\
\hline & 15 & $3.01 \pm 0.36 \mathrm{~b}$ & $5.05 \pm 0.64 \mathrm{a}$ & $5.68 \pm 0.75 a$ \\
\hline & 30 & $2.97 \pm 0.61 \mathrm{~b}$ & $5.29 \pm 0.94 \mathrm{a}$ & $5.84 \pm 0.90 \mathrm{a}$ \\
\hline & 60 & $3.39 \pm 0.67 \mathrm{ab}$ & - & - \\
\hline & 90 & $3.69 \pm 0.72 \mathrm{a}$ & - & - \\
\hline
\end{tabular}

a Values with the different letters in each column showed a statistically significant difference $(\mathrm{P}<0.05 ; \mathrm{n}=3)$; GAE, gallic acid equivalents; one-way analysis of variance followed by Duncan's post-hoc test.

Since that extraction time represents an important factor that should be optimized with the aim to minimize energy cost, the maceration was carried out using five levels of the mentioned parameter $(5,15,30,60$ and $90 \mathrm{~min})$. According to the data presented in Table 1 , the polyphenol content was increasing continuously with the time, which was in agreement with the literature (Vuleta et al., 2012; Zdravković et al., 2012). Namely, based on Fick's second law of diffusion (the extraction equilibrium will be achieved after a certain time), prolonged extraction time induces increment of polyphenol solubility, thus better polyphenol release (Jovanović et al., 2016). However, further prolongation of maceration (after $90 \mathrm{~min}$ ) did not cause statistically significant increase in total polyphenol yield (data not shown).

The influence of different particle size, solvent-to-solid ratio and extraction time levels (in maceration) on ABTS and DPPH radical scavenging activity of wild thyme extracts is shown in Figure 1.

The mathematical relationship between dependent variable (ABTS and DPPH radical scavenging activity, $\mathrm{Y}_{\mathrm{ABTS}}$ and $\left.\mathrm{Y}_{\mathrm{DPPH}}\right)$ and independent variables (particle size- $\mathrm{X}_{1}$, solventto-solid ratio- $X_{2}$ and extraction time- $X_{3}$ ), as well as their interactions, in maceration, are presented through the Equations 1 and 2

$$
\begin{gathered}
Y_{A B T S}=4.05-0.752 X_{1}+0.034 X_{1}^{2}+0.007 X_{12} \\
Y_{D P P H}=32.21-3.93 X_{1}+0.1899 X_{1}^{2}+1.08 X_{3}+0.03 X_{13}
\end{gathered}
$$

As can be seen from Figure 1 and Eq. 1 and 2, both antioxidant assays showed that particle size had a significant impact on the antioxidant activity of wild thyme extract obtained using maceration; linear term of particle size $\left(X_{1}\right)$ had a negative influence on the antioxidant activity, whereas the quadratic term of particle size $\left(\mathrm{X}_{1}^{2}\right)$ had a positive influence. The negative influence of linear term and positive influence of quadratic term of particle size mean that antioxidant activity decreased with particle size increase to a certain point, after which an increase of particle size has a positive impact on antioxidant recovery. Hydro-soluble polyphenols were mainly located in cell vacuoles, while most hydrophobic polyphenols were found in the cell wall with proteins and polysaccharides (Naczk and Shahidi, 2006), thus higher level of plant material fragmentation was needed for significant antioxidant activity against free radicals. Furthermore, with the increase of solvent-to-solid ratio, antioxidant capacity against ABTS and DPPH radicals

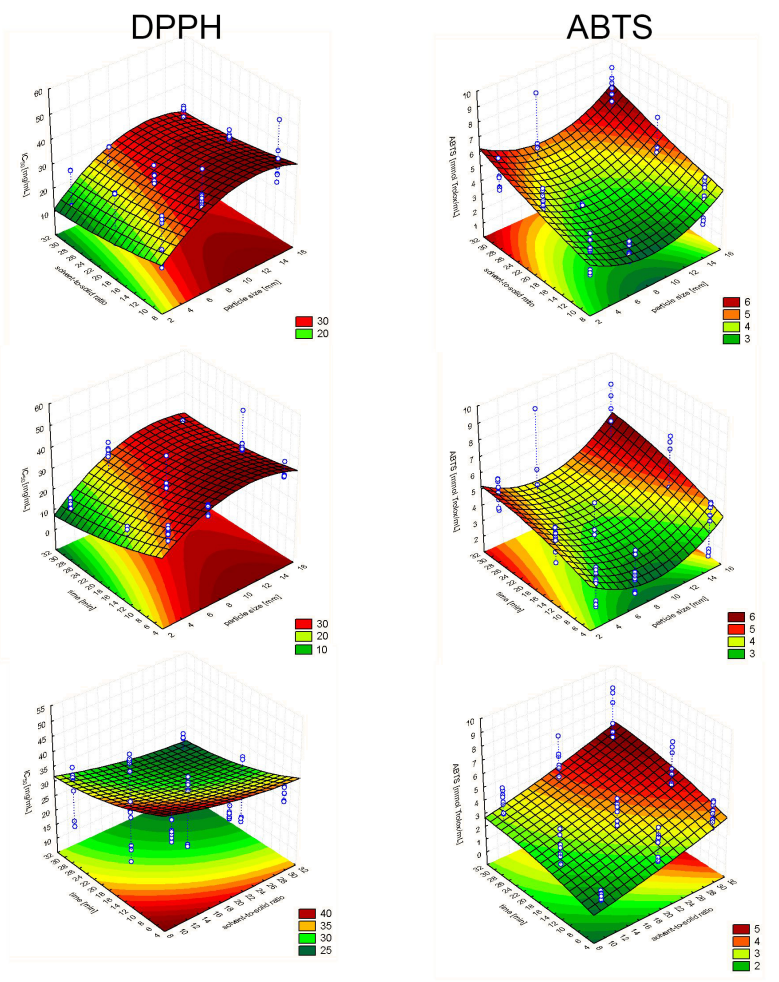

Fig. 1. Surface 3D plots of the influence of extraction conditions (particle size, solvent-to-solid ratio and extraction time) on ABTS and DPPH radical scavenging activity of wild thyme extracts, in maceration.

did not increase (Figure 1, Eq. 1 and 2). Apart from that, prolonged maceration time caused slight increase of DPPH antioxidant capacity of wild thyme extracts in both assays (Figure 1, Eq. 2) that was in agreement with total polyphenol content (Table 1). As can be seen from Eq. 1, there was a significant interaction between particle size and solvent-tosolid ratio, which indicated that the effect of the particle size on ABTS antioxidant activity was not the same at all solventto-solid ratios. Moreover, there was a significant interaction between particle size and extraction time in DPPH antioxidant activity (Eq. 2). 


\subsection{Heat-assisted extraction}

As can be seen in Table 1, the polyphenol yield was gradually enhanced with an increase in the degree of fragmentation of the herbal drug. Therefore, the highest content of polyphenols was achieved using a particle size of $0.3 \mathrm{~mm}$, while the lowest was obtained by the extraction of $1.5 \mathrm{~mm}$ diameter particles. According to Bucić-Kojić et al. (2007), there is a synergistic effect of high temperature and level of material defragmentation on extraction kinetics and yields.

Furthermore, the same trend in gradually enhanced polyphenol yield with the increase of the solvent-to-solid ratio (Table 1, 10:1 < 20:1 < 30:1). Namely, high temperature decreases solvent viscosity and causes faster mass transfer, polyphenols release, and consequently rapid extraction solvent saturation Thus, with the increment of the solvent-to-solid ratio, the polyphenols level in the extracts increases as well (Jovanović et al., 2017b).

The presented results of heat-assisted extraction indicate that there was no statistically significant difference among different extraction time levels (Table 1). Several studies have confirmed that there was not any significant difference between the polyphenols extracts obtained by different exposure times at high temperatures (Dent et al., 2013; Jovanović et al., 2017b; Vergara-Salinas et al., 2012). Moreover, high temperature and prolonged extraction time can induce the reduction of the total polyphenol content, since polyphenol compounds are thermosensitive, as well as susceptible to enzymatic degradation, polymerization and oxidation (Vergara-Salinas et al., 2012). When considering all the tested parameters, total polyphenol content was significantly higher in heat-assisted extraction, compared to maceration (Table 1). Thermal energy increases cell membrane permeability, causes the breakdown of secondary metabolites-herbal matrix connections, and consequently enhances mass transfer and polyphenols yield (Jovanović et al., 2017b; Vergara-Salinas et al., 2012). Moreover, higher polyphenol content was obtained after $5 \mathrm{~min}$ of the extraction at $80^{\circ} \mathrm{C}$, than after $90 \mathrm{~min}$ of maceration.

Different degrees of plant material fragmentation showed a significant influence on the ABTS and DPPH radical scavenging activity of wild thyme extracts obtained by using high temperature (Figure 2, Eq. 3 and 4).

$$
\begin{gathered}
Y_{A B T S}=1.82+0.025 X_{1}^{2}+0.304 X_{2}-0.005 X_{2}^{2}+0.006 X_{3}^{2}- \\
0.008 X_{12}-0.011 X_{13} \\
Y_{D P P H}=11.74+0.67 X_{1}
\end{gathered}
$$

According to Eq. 3, quadratic term of particle size $\left(\mathrm{X}_{1}^{2}\right)$, linear and quadratic terms of solvent-to-solid ratio $\left(X_{2}, X_{2}{ }^{2}\right)$, quadratic term of extraction time $\left(\mathrm{X}_{3}{ }^{2}\right)$, as well as interaction of particle size and solvent-to-solid ratio $\left(X_{12}\right)$ and interaction of particle size and time $\left(X_{13}\right)$ had a significant influence on ABTS antioxidant activity of wild thyme extracts. However, linear term of solvent-to-solid ratio had a positive impact on the ABTS antioxidant activity, whereas the quadratic term of particle size had a negative impact, thus antioxidant capacity increased with solvent-to-solid ratio increase to a certain point, after which an increase of solvent-to-solid ratio resulted in a negative effect on ABTS radical scavenging capacity. Again, higher levels of plant material degradation intensified scavenging activity against ABTS and DPPH radicals and thus better results were obtained using the smallest particles. In DPPH method, solvent-to-solid ratio did not show significant impact (Figure 2, Eq. 4). In DPPH assay, extraction time did not have statistically significant influence on radical scavenging capacity (Figure 2, Eq. 4), which was in correlation with total polyphenol content (Table 1). As can be seen from Eq 3 , there was a statistically significant effect of the interaction
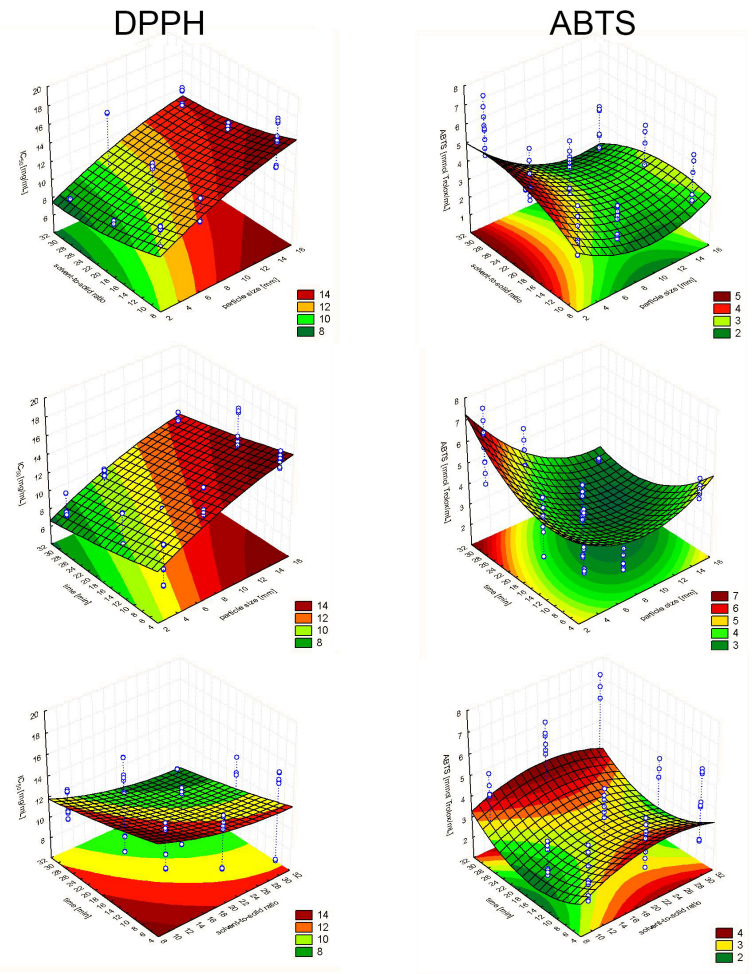

Fig. 2. Surface 3D plots of the influence of extraction conditions (particle size, solvent-to-solid ratio and extraction time) on ABTS and DPPH radical scavenging activity of wild thyme extracts, in heat-assisted extraction.

between particle size and solvent-to-solid ratio and between particle size and time, which indicated that the influence of the particle size on ABTS antioxidant activity was not the same at all solvent-to-solid and exposure time levels.

\subsection{Ultrasound-assisted extraction}

The highest total polyphenol amount was achieved using ultrasound-assisted extraction of $0.3 \mathrm{~mm}$ diameter particles, whereas the lower extraction yield was obtained using larger particles. Sun et al. (2011) also showed that the polyphenol yields in ultrasound extraction increased with the particle size decrease. It could be assumed that plant material fragmentation, together with the destructive effects of ultrasound could cause higher polyphenol release (Jovanović et al., 2017b; Sun et al., 2011).

According to the presented results (Table 1), polyphenol content in the extracts obtained in ultrasound-assisted extraction rose with the increase of the solvent-to-solid ratio, except that there was no difference between 20:1 and 30:1 ratios, suggesting that equilibrium was reached with the former. Previous studies showed that total polyphenols yield increased quickly with the increase of solvent-to-solid ratio, using the same extraction method (Bucić-Kojić et al., 2007; Galvan d'Alessandro et al., 2012; Hammi et al., 2015; Sun et al., 2011). Ultrasound waves induce degradation of plant cell walls, decrease of particle size and the enhancement of contact surface, which lead the higher mass transfer rate and faster saturation of solvent (Galvan d'Alessandro et al., 2012; Hammi et al., 2015; Jovanović et al., 2016; Sun et al., 2011). On the other hand, in the viscous extraction surrounding (the presence of large amounts of herbal drug, at 10:1 ratio) the expansion of ultrasound waves and diffusion of polyphenols were inhibited (Jovanović et al., 2017b).

Similar to the extraction at high temperature, there was no statistically significant difference between different exposure times in ultrasound-assisted polyphenol extraction (Table 1). 
Sun et al. (2011) found that there were no observable polyphenol yield changes when the extraction time was prolonged. Higher total polyphenol content in the extracts obtained using ultrasound-assisted extraction and shorter exposure time periods, in comparison to traditional extraction procedures, was previously demonstrated (Jovanović et al., 2016; Sun et al., 2011). Apart from that, prolonged ultrasound waves' exposure can cause a decrease of polyphenol yield, because of the active compounds degradation and the production of free radicals (Jovanović et al., 2016).

When other parameters were varied, polyphenol yield was significantly higher after ultrasound-assisted extraction, in comparison to the extraction at room temperature (Table 1). As can be seen from Table 1, a greater total polyphenols amount was obtained after $5 \mathrm{~min}$ of the extraction using ultrasound probe, compared to all investigated maceration times. According to the literature, ultrasound-assisted extraction provides higher yield of polyphenols during shorter exposure time, in comparison to the conventional extraction techniques (Sun et al., 2011; Zdravković et al., 2012). On the one hand, the ultrasonic waves cause extensive destruction of plant cells and release of cell content, while on the other, penetration of solvent induces the enhancement of molecule mass transfer and acceleration of the extraction process (Galvan d'Alessandro et al., 2012; Hammi et al., 2015).

In DPPH test, it was shown that particle size significantly affected radical scavenging capacity; using higher defragmentation levels of material gave better results when considering antioxidant activity (Figure 3, Eq. 6).

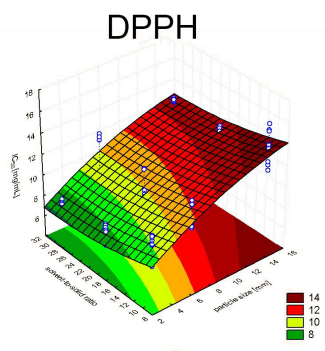

ABTS
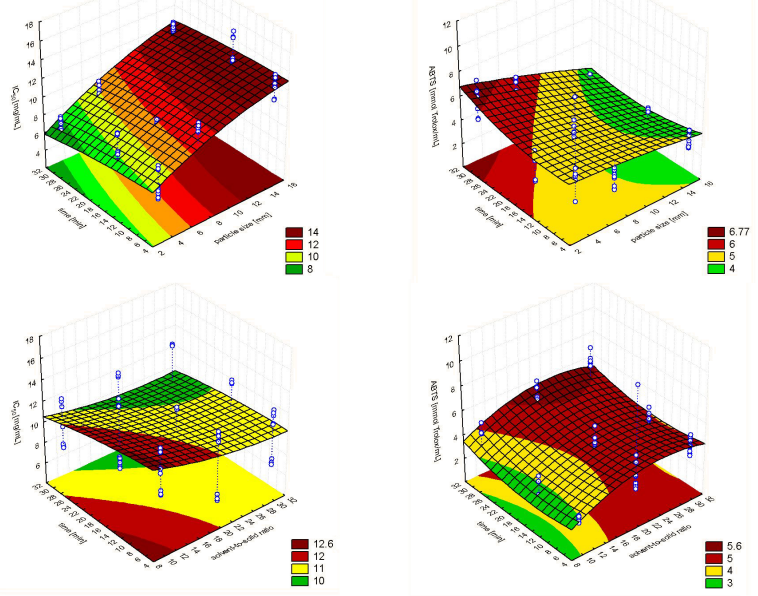

Fig. 3. Surface 3D plots of the influence of extraction conditions (particle size, solvent-to-solid ratio and extraction time) on ABTS and DPPH radical scavenging activity of wild thyme extracts, in ultrasound-assisted extraction.

$$
\begin{aligned}
Y_{A B T S} & =-0.6+0.462 X_{2}-0.009 X_{2}^{2}+0.003 X_{3}^{2} \\
& -0.006 X_{12}-0.005 X_{13} \\
Y_{D P P H} & =10.69+0.59 X_{1}-0.015 X_{1}^{2}-0.258 X_{2}
\end{aligned}
$$

According to Eq. 5, linear and quadratic terms of solvent-tosolid ratio $\left(X_{2}, X_{2}{ }^{2}\right)$, quadratic term of extraction time $\left(X_{3}{ }^{2}\right)$, as well as interaction between particle size and solvent-tosolid ratio $\left(\mathrm{X}_{12}\right)$ and interaction between particle size and time $\left(\mathrm{X}_{13}\right)$ had a significant impact on ABTS antioxidant capacity. However, in DPPH assay, only linear and quadratic terms of particle size $\left(\mathrm{X}_{1}, \mathrm{X}_{1}^{2}\right)$ and linear term of solvent-to-solid ratio $\left(X_{2}\right)$ have shown significant influence on radical scavenging capacity. Regarding the quantity of extraction medium in ultrasound-assisted extraction, with the increase of solvent quantity ABTS and DPPH antioxidant capacity increased as well (Figure 3, Eq. 5 and 6). It can be noted that linear term of solvent-to-solid ratio had a positive effect on the ABTS radical scavenging antioxidant capacity, while the quadratic term has shown a negative impact (Eq. 5); antioxidant recovery enhanced with the increase of solvent-to-solid ratio, but only to a certain point, after which an increase of solvent-tosolid ratio has caused a decrease of ABTS antioxidant activity. The positive impact of linear term and negative influence of quadratic term of particle size on DPPH antioxidant activity (Eq. 6) mean that antioxidant capacity increased with particle size increase to a certain point; after that further particle size increase has caused lower antioxidant capacity. In DPPH assay, the exposure time did not have significant impact on antioxidant activity of wild thyme extracts (Figure 3, Eq. 6), which was in correlation with total polyphenol content (Table 1). Furthermore, prolonged ultrasound extraction time can lower the antioxidant capacity of extracts, due to free radicals production by the ultrasonic waves (Jovanović et al., 2017b). According to Vergara-Salinas et al. (2012), long exposure to ultrasound waves can reduce yield of antioxidant compounds. As can be seen from Eq. 5, there was a statistically significant impact of the interaction between particle size and solvent-tosolid ratio and between particle size and extraction time, in ABTS antioxidant activity. As can be seen from Figure 2, the extracts obtained by maceration and ultrasound-assisted extraction possessed the best ABTS radical scavenging capacity. According to DPPH assay, better antioxidant effect reached in ultrasound-assisted extraction (compared to maceration and heat-assisted extraction) could be explained by the mechanism of ultrasound waves that included excessive degradation of the cell walls and decrease of the particle size, as well as higher release of the antioxidant polyphenols into the extraction medium (Galvan d'Alessandro et al., 2012; Hammi et al., 2015; Jovanović et al., 2017b). Likely, the compounds responsible for DPPH neutralization might be bound to plant cell walls, so the assistance of ultrasound waves may increase their concentration in the extracts. whereas the antioxidants for ABTS radical scavenging activity can be released without the assistance of ultrasound waves and moreover, the exposure of sonication can damage already extracted polyphenols antioxidants (Jovanović et al., 2017b).

\section{CONCLUSION}

In the present study, the impact of different particle size, solvent-to-solid ratio and extraction time levels on the polyphenol extraction from wild thyme using three extraction procedures (maceration, heat- and ultrasound-assisted extraction) was evaluated. According to the total polyphenols yield, the presented data indicated that particle size, solvent-to-solid ratio and extraction technique have shown statistically significant effect, whereas the exposure time was shown not to be a significant factor. In all three extraction procedures, the best polyphenols yield was achieved by using the smallest particles and the highest solvent-to-solid ratio. Moreover, polyphenols yields were significantly better after ultrasound-assisted extraction, in comparison to the extraction at room and high temperature. The antioxidant activity varied depending on 


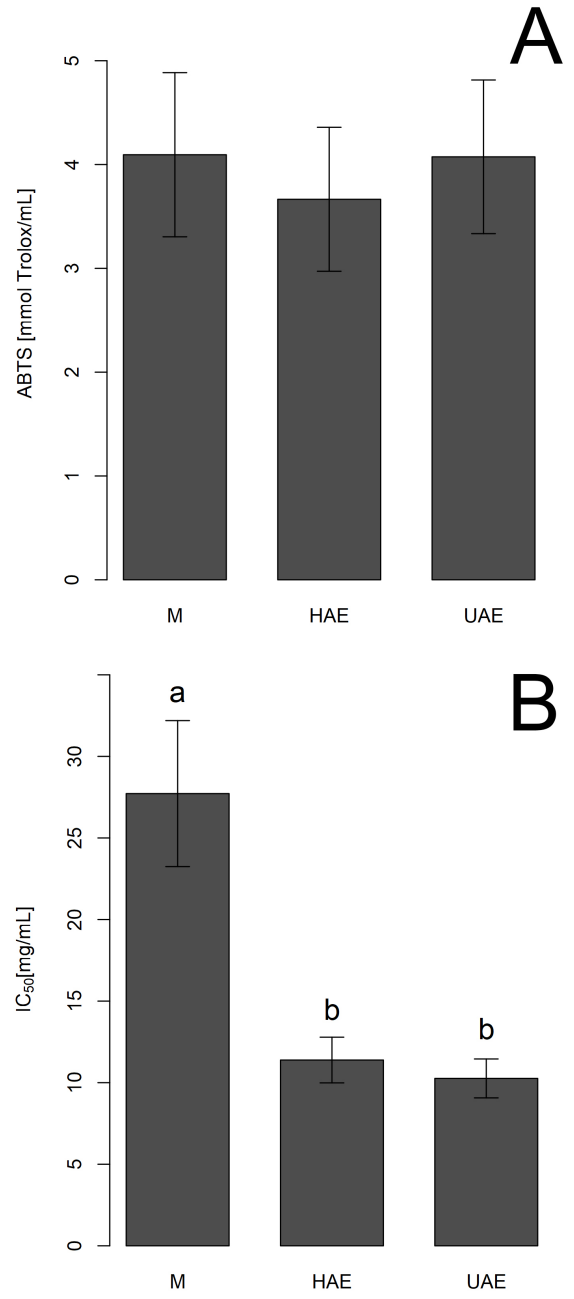

Fig. 4. Mean plots of the influence of different extraction procedures; $\mathrm{M}$ - maceration, HAE - heat-assisted extraction and UAE ultrasound-assisted extraction on ABTS (A) and DPPH (B) radical scavenging activity of wild thyme extracts; $\mathrm{IC}_{50}$, the concentration of extract required to scavenge $50 \%$ of free DPPH radicals; different letters denote statistically significant difference among mean values according to Tuckey HSD post-hoc test at level $\mathrm{P}<0.05$.

different extraction parameters and applied antioxidant assays. In ABTS test, particle size did not have significant impact on the antioxidant capacity of wild thyme extract obtained using maceration, whereas, in DPPH assay with the decrease of particle size, the antioxidant activity increased, in all three extraction techniques. With the increase of solvent-to-solid ratio ABTS antioxidant capacity increased as well, whereas in DPPH radical scavenging activity, the same parameter did not have a statistically significant influence. In maceration, prolonged extraction time has caused slight increase of ABTS and DPPH antioxidant capacity. Furthermore, in both antioxidant assays, exposure time did not have regular and significant influence on antioxidant capacity of wild thyme extracts obtained by using heat- and ultrasound-assisted extraction, which was in agreement with the total polyphenols yield. ABTS radical scavenging capacity of various wild thyme extracts followed the next trend: maceration and ultrasound-assisted extraction $>$ heat-assisted extraction, and in opposite, in DPPH neutralization: ultrasound-assisted extraction > heat-assisted extraction » maceration. The study represents an important step in formulation of antioxidant-rich extracts with potential use in food, pharmaceutical, and cosmetic industry.

\section{ACKNOWLEDGMENTS}

The authors acknowledge their gratitude to the Ministry of Education, Science and Technological Development of Serbia, project numbers 46010 and 46013.

\section{REFERENCES}

Boros, B., Jakabová, S., Dörnyei, g., Horváth, G., Pluhár, Z., Kilár, F. and Felinger, A. (2010). Determination of polyphenolic compounds by liquid chromatography-mass spectrometry in Thymus species, Journal of Chromatography A 1217(51): 7972-7980.

Bucić-Kojić, A., Planinić, M., Tomas, S., Bilić, M. and Velić, D. (2007). Study of solid-liquid extraction kinetics of total polyphenols from grape seeds, Journal of Food Engineering 81(1): 236-242.

Čanadanović-Brunet, J. M., Đilas, S. M., Ćetković, G. S., Tumbas, V. T., Mandicć, A. I. and Čanadanović, V. M. (2006). Antioxidant activities of different Teucrium montanum L. extracts, International Journal of Food Science and Technology 41(6): 667-673.

Casiglia, S., Bruno, M., Scandolera, E., Senatore, F. and Senatore, F. (2015). Influence of harvesting time on composition of the essential oil of Thymus capitatus (L.) Hoffmanns. \& Link. growing wild in northern Sicily and its activity on microorganisms affecting historical art crafts, Arabian Journal of Chemistry p. S1878535215001653.

Dent, M., Dragović-Uzelac, V., Penić, M., Bosiljkov, T. and Levaj, B. (2013). The effect of extraction solvents, temperature and time on the composition and mass fraction of polyphenols in Dalmatian wild sage (Salvia officinalis L.) extracts, Food Technology and Biotechnology 51(1): 84-91.

Galvan d'Alessandro, L., Kriaa, K., Nikov, I. and Dimitrov, K. (2012). Ultrasound assisted extraction of polyphenols from black chokeberry, Separation and Purification Technology 93: 42-47.

González, E., Gómez-Caravaca, A. M., Giménez, B., Cebrián, R., Maqueda, M., Martínez-Férez, A., Segura-Carretero, A. and Robert, P. (2019). Evolution of the phenolic compounds profile of olive leaf extract encapsulated by spray-drying during in vitro gastrointestinal digestion, Food Chemistry 279: $40-48$.

Hammi, K. M., Jdey, A., Abdelly, C., Majdoub, H. and Ksouri, R. (2015). Optimization of ultrasound-assisted extraction of antioxidant compounds from Tunisian Zizyphus lotus fruits using response surface methodology, Food Chemistry 184: 8089.

Hosseinzadeh, S., Kukhdan, A. J., Hosseini, A. and Armand, R. (2015). The application of Thymus vulgaris in traditional and modern medicine: a review, Global Journal of Pharmacology 9(3): 260-266.

Jovanović, A. A., Đorđević, V. B., Zdunić, G. M., Pljevljakušić, D. S., Šavikin, K. P., Gođevac, D. M. and Bugarski, B. M. (2017a). Optimization of the extraction process of polyphenols from Thymus serpyllum L. herb using maceration, heatand ultrasound-assisted techniques, Separation and Purification Technology 179: 369-380.

Jovanović, A., Đorđević, V., Zdunić, G., Šavikin, K., Pljevljakušić, D. and Bugarski, B. (2016). Ultrasound-assisted extraction of polyphenols from Thymus serpyllum and its antioxidant activity, Hemijska industrija 70(4): 391-398. 
Jovanović, A., Petrović, P., Đorđevic, V., Zdunić, G., Šavikin, K. and Bugarski, B. (2017b). Polyphenols extraction from plant sources, Lekovite sirovine 37: 45-49.

Naczk, M. and Shahidi, F. (2006). Phenolics in cereals, fruits and vegetables: Occurrence, extraction and analysis, Journal of Pharmaceutical and Biomedical Analysis 41(5): 1523-1542.

Nikolić, M., Glamočlija, J., Ferreira, I. C., Calhelha, R. C., Fernandes, n., Marković, T., Marković, D., Giweli, A. and Soković, M. (2014). Chemical composition, antimicrobial, antioxidant and antitumor activity of Thymus serpyllum L., Thymus algeriensis Boiss. and Reut and Thymus vulgaris L. essential oils, Industrial Crops and Products 52: 183-190.

Petrović, P., Ivanović, K., Jovanović, A., Simović, M., Milutinović, V., Kozarski, M., Petković, M., Cvetković, A., Klaus, A. and Bugarski, B. (2019). The impact of puffball autolysis on selected chemical and biological properties: Puffball extracts as potential ingredients of skin-care products, Archives of Biological Sciences 71(4): 721-733.

Petrović, P., Vunduk, J., Klaus, A., Kozarski, M., Nikšić, M., Žižak, Ž., Vuković, N., Šekularac, G., Drmanić, S. and Bugarski, B. (2016). Biological potential of puffballs: A comparative analysis, Journal of Functional Foods 21: 36-49.

Skotti, E., Anastasaki, E., Kanellou, G., Polissiou, M. and Tarantilis, P. A. (2014). Total phenolic content, antioxidant activity and toxicity of aqueous extracts from selected Greek medicinal and aromatic plants, Industrial Crops and Products 53: $46-54$.

Spormann, T. M., Albert, F. W., Rath, T., Dietrich, H., Will, F., Stockis, J.-P., Eisenbrand, G. and Janzowski, C. (2008). Anthocyanin/polyphenolic-rich fruit juice reduces oxidative cell damage in an intervention study with patients on hemodialysis, Cancer Epidemiology Biomarkers $\mathcal{E}$ Prevention 17(12): 3372-3380.

Sun, Y., Liu, Z. and Wang, J. (2011). Ultrasound-assisted extraction of five isoflavones from Iris tectorum Maxim, Separation and Purification Technology 78(1): 49-54.

Vergara-Salinas, J. R., Pérez-Jiménez, J., Torres, J. L., Agosin, E. and Pérez-Correa, J. R. (2012). Effects of temperature and time on polyphenolic content and antioxidant activity in the pressurized hot water extraction of deodorized thyme (Thymus vulgaris), Journal of Agricultural and Food Chemistry 60(44): 10920-10929.

Vuleta, G., Milić, J. and Savić, S. (eds) (2012). Farmaceutska tehnologija [Pharmaceutical technology], Faculty of Pharmacy, University of Belgrade, Belgrade, Serbia.

Zdravković, A., Stanojević, L., Stanković, M., Cakić, M., Nikolić, V., Nikolić, L. and Ilić, D. (2012). The influence of operational conditions and the extraction techniques on the yield, kinetics and composition of aqueous-ethanolic extracts from the nettle leaf (Urtica dioica 1.), Savremene tehnologije 1(1): 30-37. 